\title{
Supporting Information: Enhanced nonlinear light generation in oligomers of silicon nanoparticles under vector beam illumination
}

\author{
Maria K. Kroychuk ${ }^{1}$, Alexander S. Shorokhov ${ }^{1}$, Damir F. Yagudin ${ }^{1}$, Daniil \\ A. Shilkin ${ }^{1}$, Daria A. Smirnova ${ }^{2,3}$, Irina Volkovskaya ${ }^{3}$, Maxim R. \\ Shcherbakov ${ }^{4,1}$, Gennady Shvets ${ }^{4}$, and Andrey A. Fedyanin ${ }^{1}$ \\ ${ }^{1}$ Faculty of Physics, Lomonosov Moscow State University, Moscow 119991, \\ Russia \\ ${ }^{2}$ Nonlinear Physics Centre, Australian National University, Canberra, ACT \\ 2601, Australia \\ ${ }^{3}$ Institute of Applied Physics, Nizhny Novgorod 603950, Russia \\ ${ }^{4}$ School of Applied and Engineering Physics, Cornell University, Ithaca, NY \\ 14853, USA
}

Section 1: sample fabrication. An amorphous silicon film was deposited on a 170um-thick coverslip fused silica glass; the thickness of the film was determined to be $260 \pm$ $5 \mathrm{~nm}$ with a spectroscopic ellipsometer. A 100-nm-thick hydrogen silsesquioxane (HSQ) e-beam resist film was spun on the a-Si film and baked at $170^{\circ} \mathrm{C}$ for $2 \mathrm{~min}$. The pattern, which included trimers, quadrumers and monomers of different diameters and interparticle spacing, was exposed at $700 \mu \mathrm{C} / \mathrm{cm}^{2}$. The mask was developed in AZ $300 \mathrm{MIF}$ developer for $90 \mathrm{~s}$, rinsed in DI water for $60 \mathrm{~s}$ and blown dry with nitrogen. The pattern was transferred to the silicon layer using deep reactive-ion etching in a fluorine-based plasma (Unaxis SLR 770) using a mixture of $\mathrm{SF}_{6}$ and $\mathrm{C}_{4} \mathrm{~F}_{8}$ to provide smooth vertical sidewalls, at an etch rate of $100 \mathrm{~nm} / \mathrm{min}$. The remainder of the HSQ mask was not removed after the etch step.

Section 2: modeling the excitation of the collective out-of-plane magnetic mode. The optical scattering spectra for silicon disk oligomers illuminated by azimuthally polarized cylindrical vector beams (AB) are calculated using Lumerical FDTD Solutions software package. To model AB, we used K-space beam profile definition with an approximate numerical aperture (NA). First, we define a beam envelope function as 
Gaussian profile: $E_{\text {env }}(\mathbf{k})=\exp \left(-\frac{k_{x}^{2}+k_{y}^{2}}{2(k \times N A)^{2}}\right)$, where $k=2 \pi / \lambda$. Then we define electric and magnetic field vector components using slightly modified formulas for the azimuthally polarized beam from [1]:

$$
\begin{gathered}
E_{x}(\mathbf{k})=-\sin (\varphi) \times E_{\text {env }}(\mathbf{k}), H_{x}(\mathbf{k})=-\sqrt{\frac{\varepsilon_{0}}{\mu_{0}}} \cos (\varphi) \times \cos (\theta) \times E_{\text {env }}(\mathbf{k}) \\
E_{y}(\mathbf{k})=-\cos (\varphi) \times E_{\text {env }}(\mathbf{k}), H_{y}(\mathbf{k})=-\sqrt{\frac{\varepsilon_{0}}{\mu_{0}}} \sin (\varphi) \times \cos (\theta) \times E_{\text {env }}(\mathbf{k}) \\
E_{z}(\mathbf{k})=0, H_{z}(\mathbf{k})=\sqrt{\frac{\varepsilon_{0}}{\mu_{0}}} \sin (\theta) \times E_{\text {env }}(\mathbf{k})
\end{gathered}
$$

Next, we obtain these vector components in real space using chirp Z-transform and normalize fields, so that electric field amplitude equals unity and repeat the procedure for different values of $\lambda$ in desired range to create a broadband source. To calculate the scattering cross-section, the total field/scattered field technique was utilized. The scattered field was obtained in two consecutive steps: first, we performed the computation without the scattering object to get incident field components in the area around the scatterer, then we repeated the previous step with a cluster present in the calculation area to obtain the total field. Scattered field is then derived by using the standard formula: $\mathbf{E}_{s c a t}=\mathbf{E}_{t o t}-\mathbf{E}_{i n c}, \mathbf{H}_{\text {scat }}=\mathbf{H}_{t o t}-\mathbf{H}_{i n c}$. By integrating the Poynting vector of the scattered field over a closed surface (in our case, a cube) surrounding the cluster and normalizing the obtained scattered power by the average incident field intensity, we obtained the total scattering cross-section.
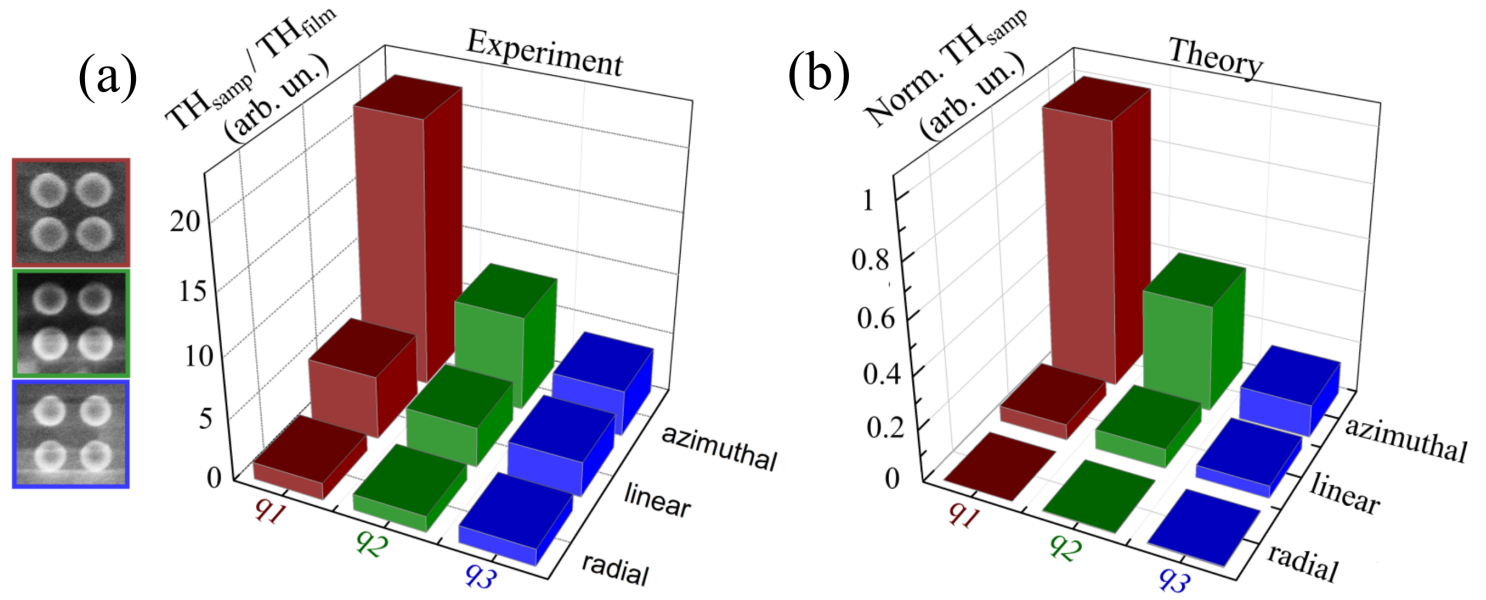

Figure S1. (a) Color columns indicate experimental THG enhancement from the isolated nanoclusters relative to the $\mathrm{TH}$ signal from an unstructured silicon film of the same hight. The clusters consist of disks with different diameters and similar interparticle distances. Panels to the left show SEM images of the oligomers understudy with colorcoded outlines matching the colors of their respective normalized TH response. (b) Numerically simulated THG enhancement for the quadrumers illuminated by AB, RB and LB. 
As described in the main part of the text, the collective out-of-plane magnetic dipoles (OMD) with an approximately $20 \mathrm{~nm}$ blue shift from the magnetic dipolar resonance (MDR) spectral position are excited under illumination by $\mathrm{AB}$ at normal incidence. Spectral shift of the collective mode, while changing the diameter of nanodisks $(\mathrm{d})$ for a fixed height $(h=260 \mathrm{~nm})$ and similar interparticle distance $(s \approx$ $100 \mathrm{~nm}$ ), manifests itself in the third-harmonic (TH) intensity variation detected in the $\mathrm{TH}$ microscopy experiments presented in Figure S1(a), when changing experimental samples sizes $\left(q_{1}: d=281 \mathrm{~nm}, s=70 \mathrm{~nm} ; q_{2}: d=254 \mathrm{~nm}, s=112 \mathrm{~nm} ; q_{3}: d=243 \mathrm{~nm}\right.$, $s=114 \mathrm{~nm})$ for the fixed fundamental wavelength $(\lambda=1050 \mathrm{~nm})$. The obtained results are confirmed numerically using the FEM method for given oligomer sizes (Fig. S1(b)).

(a)

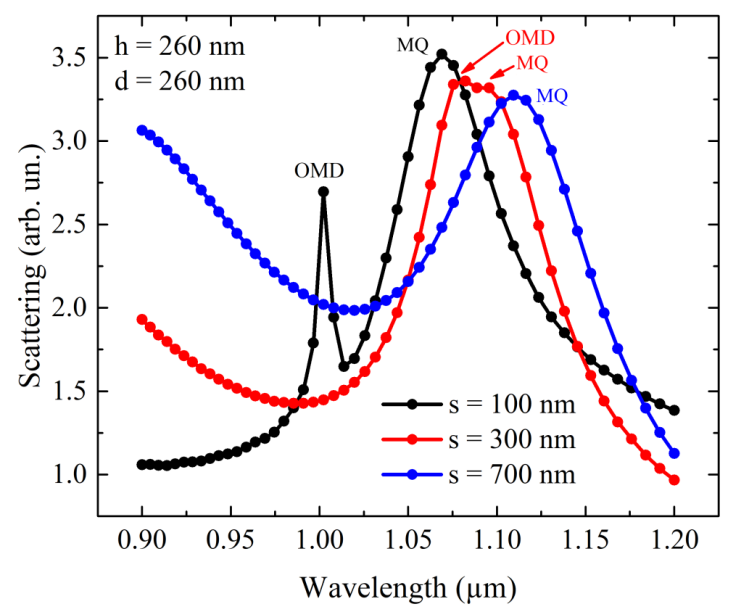

(b)

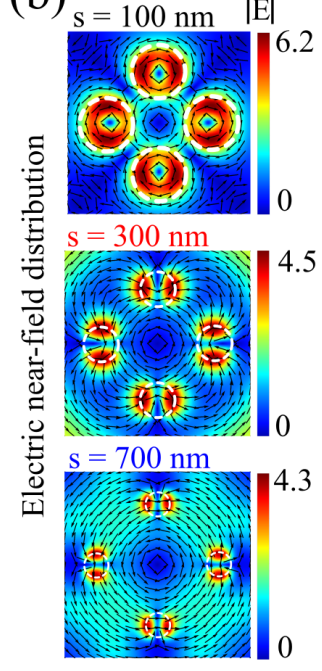

Figure S2. (a) Numerical spectra of isolated silicon quadrumer with various spacing between nanodisks for AB excitation at normal incidence. (b) Simulated electric field distributions at the center xy-cross-section of the quadrumer studied in (a) at the wavelength of $\mathrm{OMD}$ mode for $\mathrm{AB}$ excitation at normal incidence. Color indicates the normalized electric field magnitude and white dashed circles show the position of the nanodisks.

We calculate scattering of the isolated quadrumer and electric local field distributions in its cross-section parallel to the interface for the fixed height $(h=260 \mathrm{~nm})$ and diameter $(d=260 \mathrm{~nm})$ of nanoparticles, but varying interparticle distance from $\mathrm{s}=100 \mathrm{~nm}$ to $\mathrm{s}=700 \mathrm{~nm}$ (Fig. S2(a), S2(b)) to determine parameters for the OMD mode excitation. While interparticle spacing is small $(s=100 \mathrm{~nm}$, black line in Figure S2(a)), strong near-field interaction between nanodisks is observed, resulting in OMD mode excitation on the short-wavelength slope of the MDR. The electromagnetic field is tightly bound in the volume of nanodisks. With the increase of spacing between nanoparticles, interaction between them decreases and the collective mode spectrally overlaps with the MD mode (Fig. S2(a), S2(b), $s=300 \mathrm{~nm}$ ). It becomes difficult to distinguish them, especially in the linear response of oligomers. When the spacing reaches $s=500 \mathrm{~nm}$, nanodisks start to behave as independent nanoobjects. Independent MDR manifests itself as a peak in the scattering spectrum, the shape and spectral position do not change while disks move 
(a)

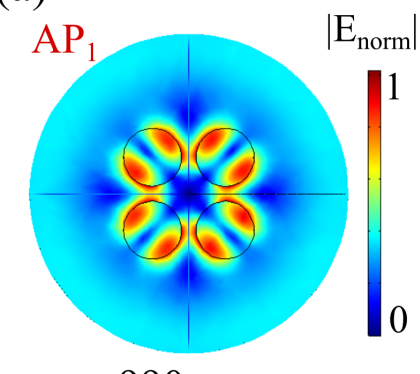

(c)

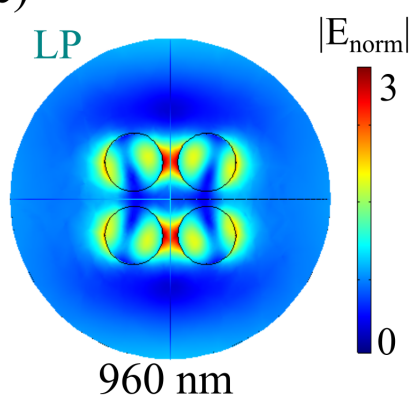

(b)

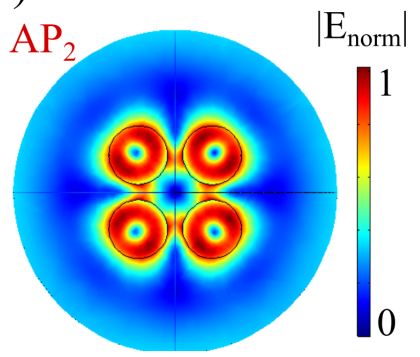

(d)

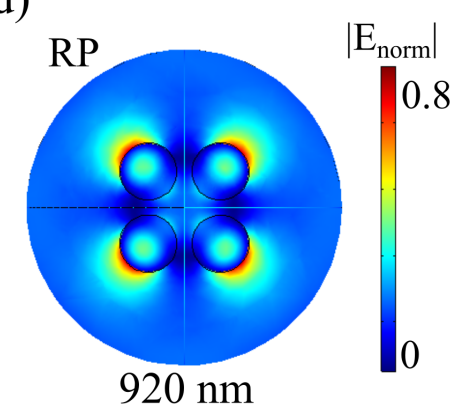

Figure S3. Simulated near-field distributions normalized to the incident field. (a) $\mathrm{AP}_{1}$ corresponds to the wavelength of the oligomer $\mathrm{MQR}$ under $\mathrm{AB}$ excitation; (b) $\mathrm{AP}_{2}$ corresponds to the OMD mode under $\mathrm{AB}$ excitation; (c) LP stands for the MDR of the oligomer under LB excitation, and (d) RP stands for the electric field distribution under RB illumination.

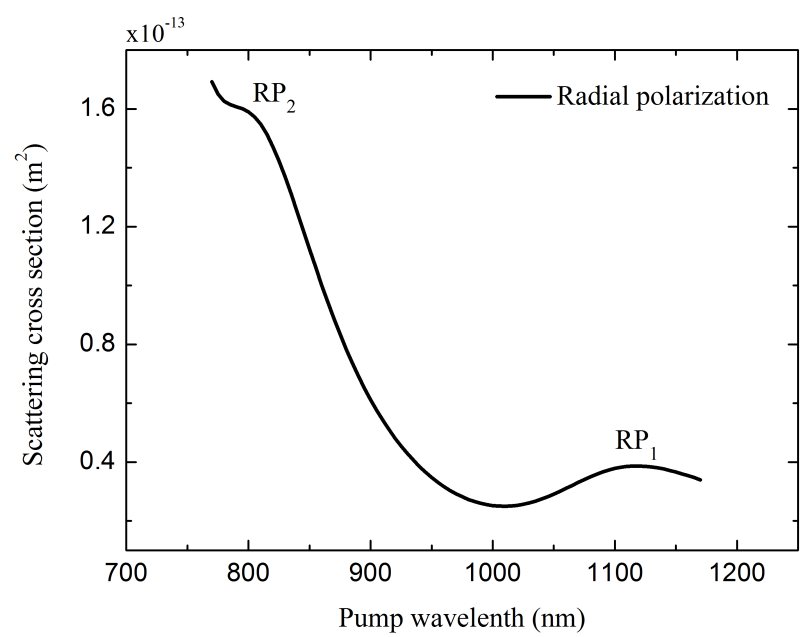

Figure S4. Numerical scattering cross section spectrum for the quadrumer of a-Si nanodisks for RB illumination at normal incidence within an extended spectral range.

apart (Fig. S2(a), blue line).

Near-field distributions in Fig. S3 indicate the excitation of distinct collective modes in the quadrumer by different pump polarizations. To supplement Fig. 2(a) of the main text, Fig. S4 presents the calculated scattering spectrum of RB in a wider wavelength range.

We calculated the $\mathrm{TH}$ intensity for an individual nanodisk and divided it by the $\mathrm{TH}$ 


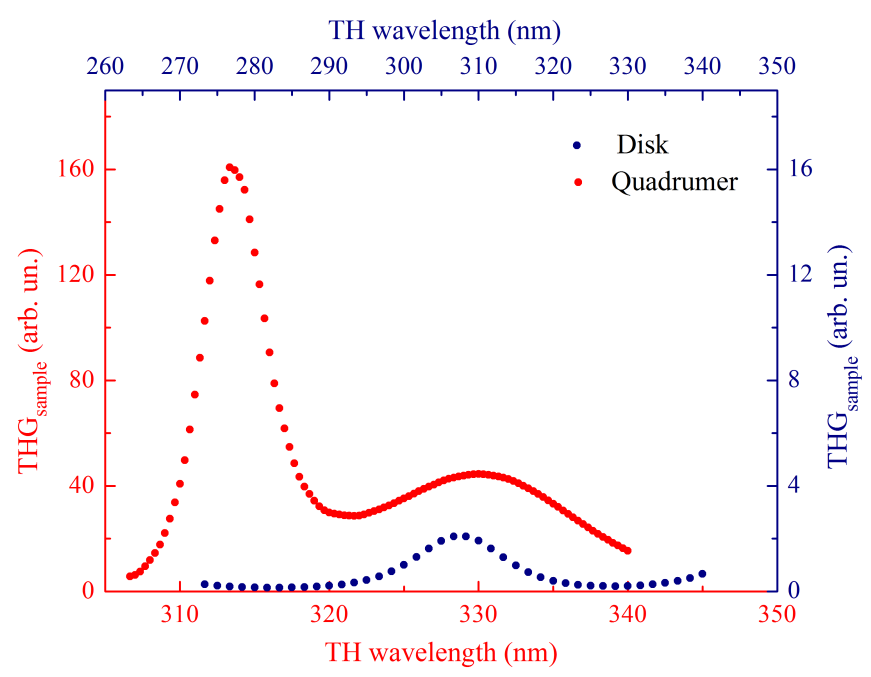

Figure S5. Numerical TH intensity spectra for the quadrumer of a-Si nanodisks (red dots) and an isolated disk (blue dots) under AB excitation.

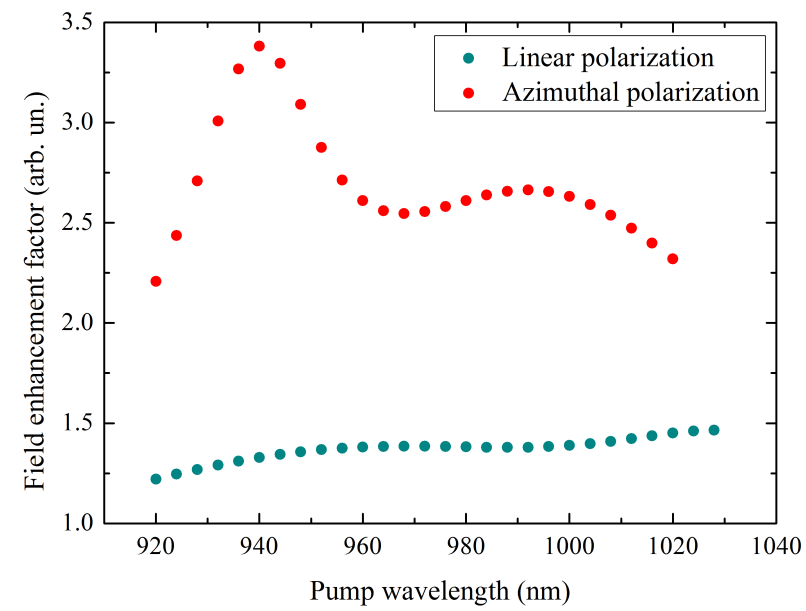

Figure S6. Numerical spectra of the field enhancement factor for an isolated quadrumer under $\mathrm{AB}$ and $\mathrm{LB}$ excitation.

intensity from an unstructured film. We compared the obtained TH enhancement with the normalized TH intensity from of an isolated oligomer (Fig. S5). For AB polarization the difference amounted to more than 81 times. The obtained numerical results prove the efficiency of utilizing the collective optical magnetic mode in order to enhance the nonlinear response of the nanosystem.

The simple coupled-mode considerations suggest scaling the THG efficiency depending on the detunings from the resonances at the fundamental and $\mathrm{TH}$ frequencies:

$$
\tilde{\rho} \sim Q_{\mathrm{FF}}^{3} Q_{\mathrm{TH}}\left(\frac{\gamma_{\mathrm{FF}}^{2}}{\left(\omega-\omega_{\mathrm{FF}}\right)^{2}+\gamma_{\mathrm{FF}}^{2}}\right)^{3} \frac{\gamma_{\mathrm{TH}}^{2}}{\left(3 \omega-\omega_{\mathrm{TH}}\right)^{2}+\gamma_{\mathrm{TH}}^{2}} .
$$

Here, $Q_{\mathrm{FF}}$ and $Q_{\mathrm{TH}}$ are quality factors of resonances, $\omega_{\mathrm{FF}}$ and $\omega_{\mathrm{TH}}$ are resonance frequencies, $\gamma_{\mathrm{FF}}$ and $\gamma_{\mathrm{TH}}$ are damping constants including the ohmic and radiative losses. 
The factor at the TH frequency is non-resonant in our case.

We also numerically studied the field enhancement factors for AB and LB quadrumer excitation and obtained that for $\mathrm{AB}$ case, it is more than twice than that for $\mathrm{LB}$ within the whole spectral range (Fig. S6).

\section{Section 3: experimental methods for measuring scattering spectra of isolated}

oligomers. The experimental setup for measuring scattering spectra is shown in Fig. S7. Light from a $50 \mathrm{~W}$ halogen lamp is collected by a plano-convex lens L1 $(f=30 \mathrm{~mm})$ and coupled into a multimode optical fiber F1 (core diameter $=0.6 \mathrm{~mm}, \mathrm{NA}=0.48$ ) by an aspheric condenser lens L2 ( $f=20 \mathrm{~mm}$, anti-reflection coating for visible light). The fiber output is collimated by an aspheric condenser lens L3 $(f=10.5 \mathrm{~mm})$. The field diaphragm FD is placed in the focal plane of an achromatic doublet L4 ( $f=250 \mathrm{~mm}$, antireflection coating for 400-1000 $\mathrm{nm}$ light) and imaged on the sample by a plan achromat strain-free objective lens $\mathrm{O} 1(f=25 \mathrm{~mm}, \mathrm{NA}=0.1-0.25)$. A Glan-Tailor prism GP controls the polarisation of the incident light. The NA was set to about 0.15 .

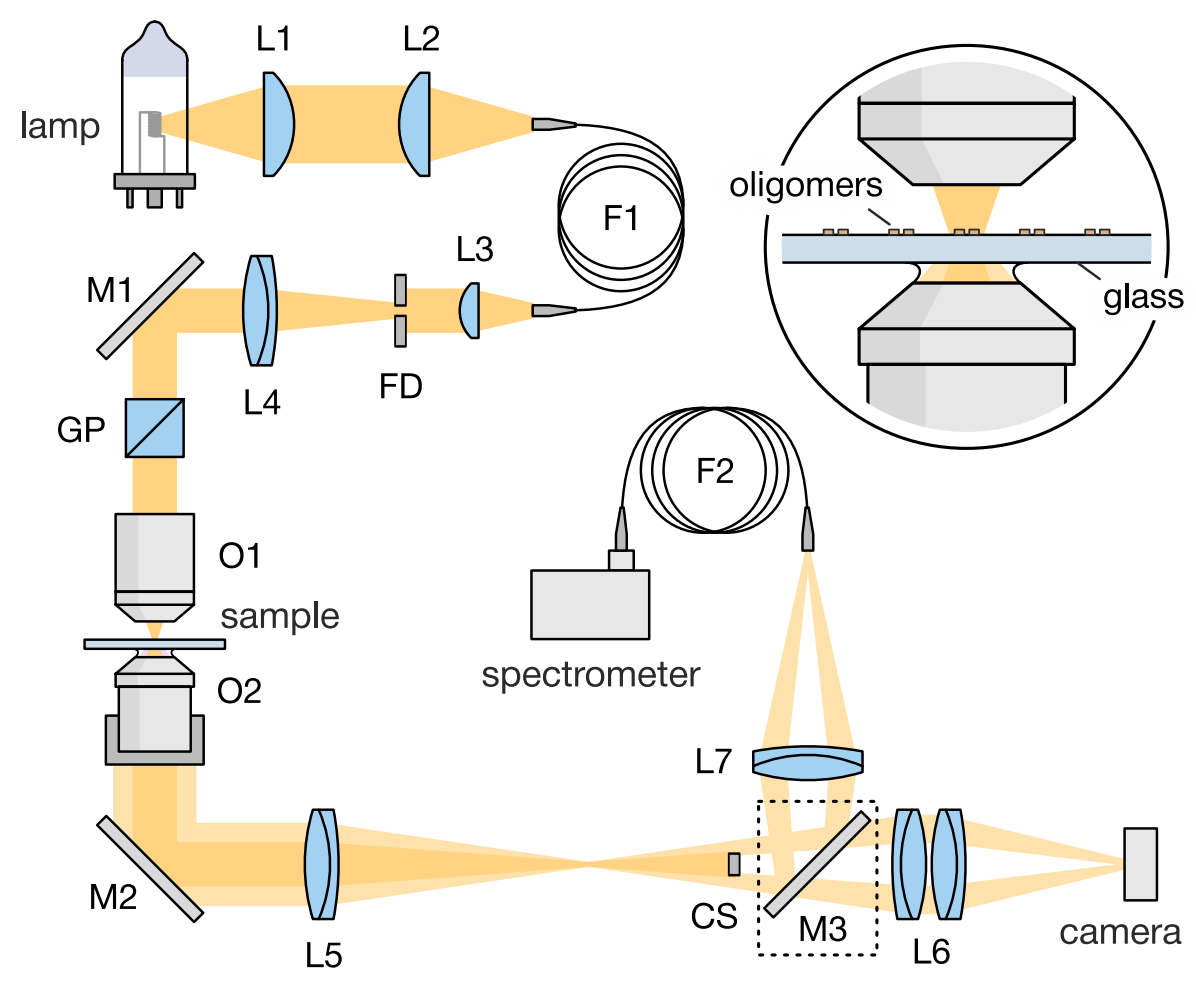

Figure S7. Experimental setup for scattering spectroscopy. L1:7 - lenses; F1,2 multimode optical fibers; FD - field diaphragm; M1:3 - silver mirrors; GP - Glan-Tailor prism; O1,2 - objective lenses; CS - central stop. Mirror M3 is removable.

The sample is placed on a two-dimensional translation stage with the structure facing upwards. A water-immersion plan apochromatic objective lens O2 (Olympus UPlanSApo $60 \mathrm{XW}$ ) with an NA of 1.2 is mounted on a vertical translation stage. An image of its back focal plane is formed on a central stop CS (diameter $1 \mathrm{~mm}$ ) in a $2 \mathrm{f}-2 \mathrm{f}$ configuration by an achromatic doublet L5 ( $f=100 \mathrm{~mm}$, anti-reflection coating for 400-1000 nm light). The central stop CS blocks unscattered illumination. 
Depending on the position of the mirror M3 on a flip mount, a dark-field image of the sample is formed either on a camera or on the facet of an optical fiber F2 connected to a spectrometer. In the first case, the sample is imaged by a couple of achromatic doublets L6 ( $f=200 \mathrm{~mm}$ and $f=60 \mathrm{~mm}$, anti-reflection coating for visible light). In the second case, the image is formed by an achromatic doublet L7 ( $f=85 \mathrm{~mm}$, anti-reflection coating for 400-1000 nm light). The fiber F2 has a core diameter of $105 \mu \mathrm{m}$, which corresponds to a sample area of approximately $3 \mu \mathrm{m}$ in diameter, and mounted on a three-dimensional translation mount. The spectrometer is an Ocean Optics QE Pro with a resolution of $2 \mathrm{~nm}$.

The scattering spectra were obtained as (Signal - Background)/(Lamp - Noise). The background was measured at a place on the sample where there are no particles.
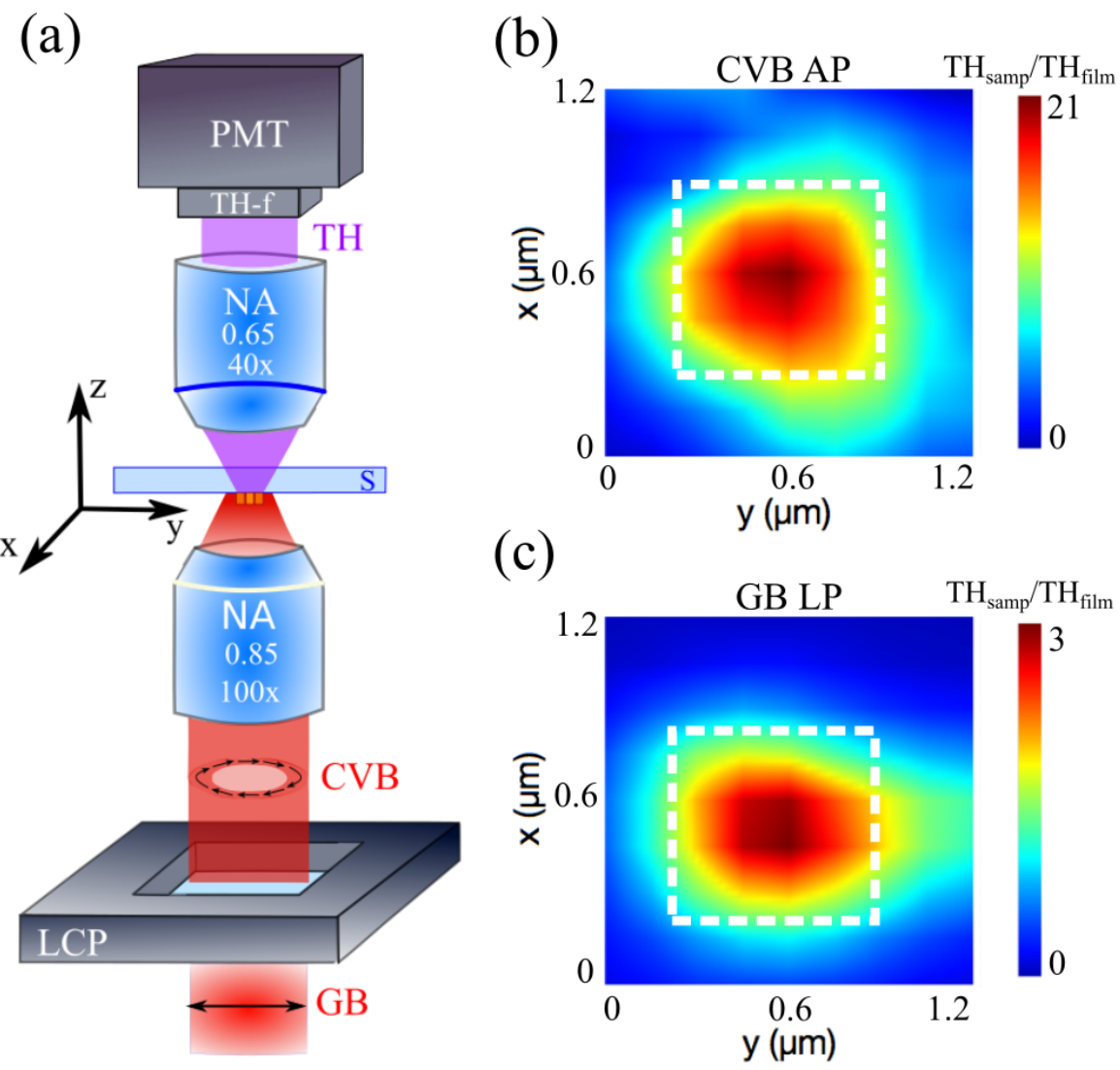

Figure S8. (a) Schematic of a main part of the TH microscopy setup with a possibility of pump polarization tuning: CVB - cylindrical vector beam (with AP and RP) and GB Gaussian beam (with LP): LCP - liquid crystal polarizer, S - sample on a three-coordinate micrometer stage with piezo control, TH-f - set of BG39 colour filters for separation of TH radiation from the pump beam, PMT - photomultiplier assembly connected to the lock-in amplifier. (b), (c) The results of the TH intensity mapping for the isolated oligomer: (b) for azimuthally polarized pump beam, (c)-for linearly polarized pump beam. The color bar indicates the gain of the $\mathrm{TH}$ intensity from the oligomer relative to the $\mathrm{TH}$ intensity from the unstructured silicon film of the same height placed into the main channel of the setup. 


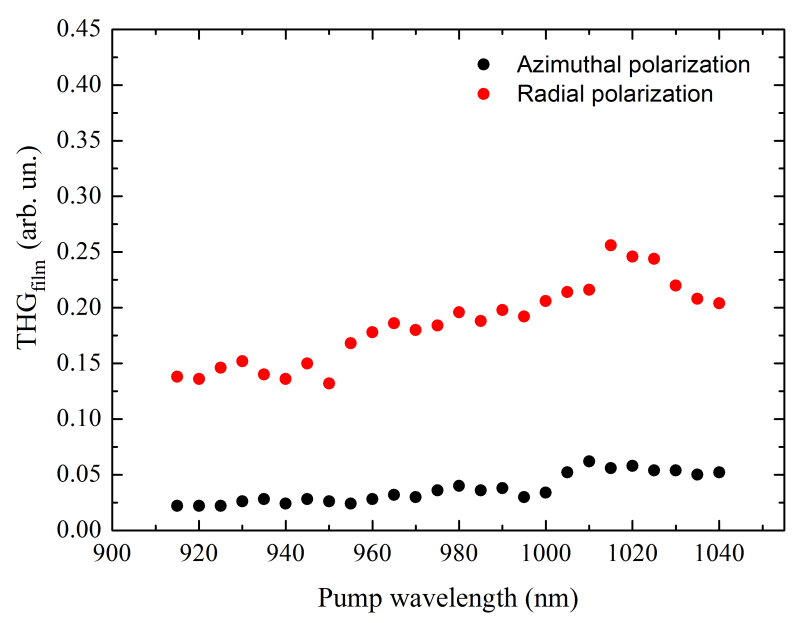

Figure S9. Experimental TH intensity spectra for an unstructured a-Si film for AB (red dots) and RB (black dots) illumination at normal incidence for fixed pump power.

\section{Section 4: experimental methods of the third-harmonic micro-spectroscopy of} isolated oligomers. Figure S8(a) shows the main part of the TH micro-spectroscopy setup described in the main part of the text. For every fixed wavelength of the pump beam from $\lambda=930 \mathrm{~nm}$ to $\lambda=1040 \mathrm{~nm}$ we repeat the same procedure: combine the laser beam spot and the focal plane of the objective lens with $\mathrm{NA}=0.85$ with the center of the oligomer, using a three-coordinate automated platform. For this purpose we match the image of the isolated sample and the image of the pump beam using the CMOS camera and increase the TH signal from the isolated oligomer, moving the whole sample mechanically in three perpendicular directions. Then we make accurate measurements of the $\mathrm{TH}$ power through the process of $2 \mathrm{~d}$ scanning of the sample with a step of $50 \mathrm{~nm}$ automated in the LabView software package. During scanning procedure, the sample is moving between two objectives in a plane perpendicular to the propagation direction of the laser pulse, gaining the maximum possible $\mathrm{TH}$ power. We also measure the pump beam power just before the objective focusing lens to control the laser sability The results of the TH microscopy for AB and LB are presented for resonant oligomer in Fig. S8(b) and $\mathrm{S} 8(\mathrm{c})$, respectively. In all experiments, the $\mathrm{TH}$ intensity from the isolated cluster is divided to the TH intensity of the Si film under the same experimental conditions (Fig. S9), also excluding the influence of the pump beam structure on the THG enhancement. Maximum of the normalized $\mathrm{TH}$ output is observed when the center of the pump beam coincides with the center of the oligomer (white dashed rectangles in Fig. S8(b), S8(c) show the positions of the samples).

We use the radial polarization converter ARCoptix with nematic liquid crystal cells to convert LB to CVBs with azimuthal or radial polarization. The polarizer used in this work consists of three parts: a cell that rotates linear polarization by $90^{\circ}$ under applied voltage, thereby making the transition from $\mathrm{AB}$ to $\mathrm{RB}$; polarization converter $(\theta$-cell), that locally rotates electric field polarization and phase compensator that removes phase difference 


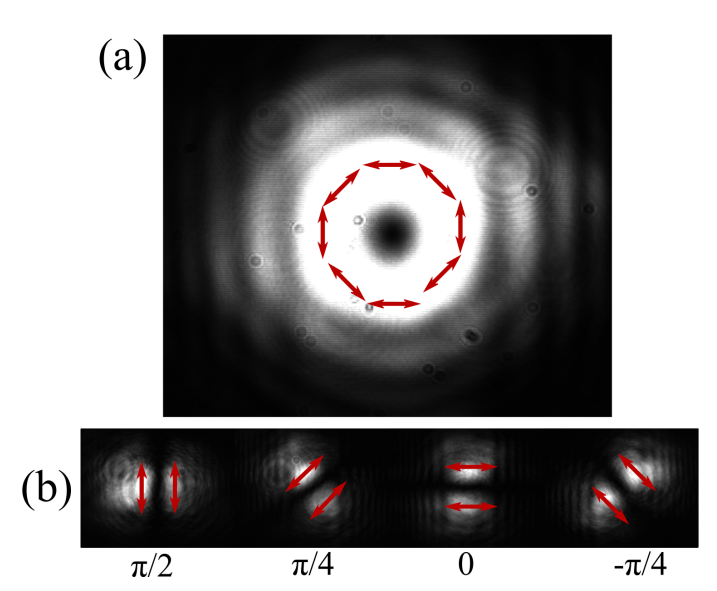

Figure S10. (a) Direct plane image of AB visualized by the CMOS camera. Arrows show the electric field polarization in different points of the beam. (b) Image of the beam on the camera after passing through the analyzer at different angles.

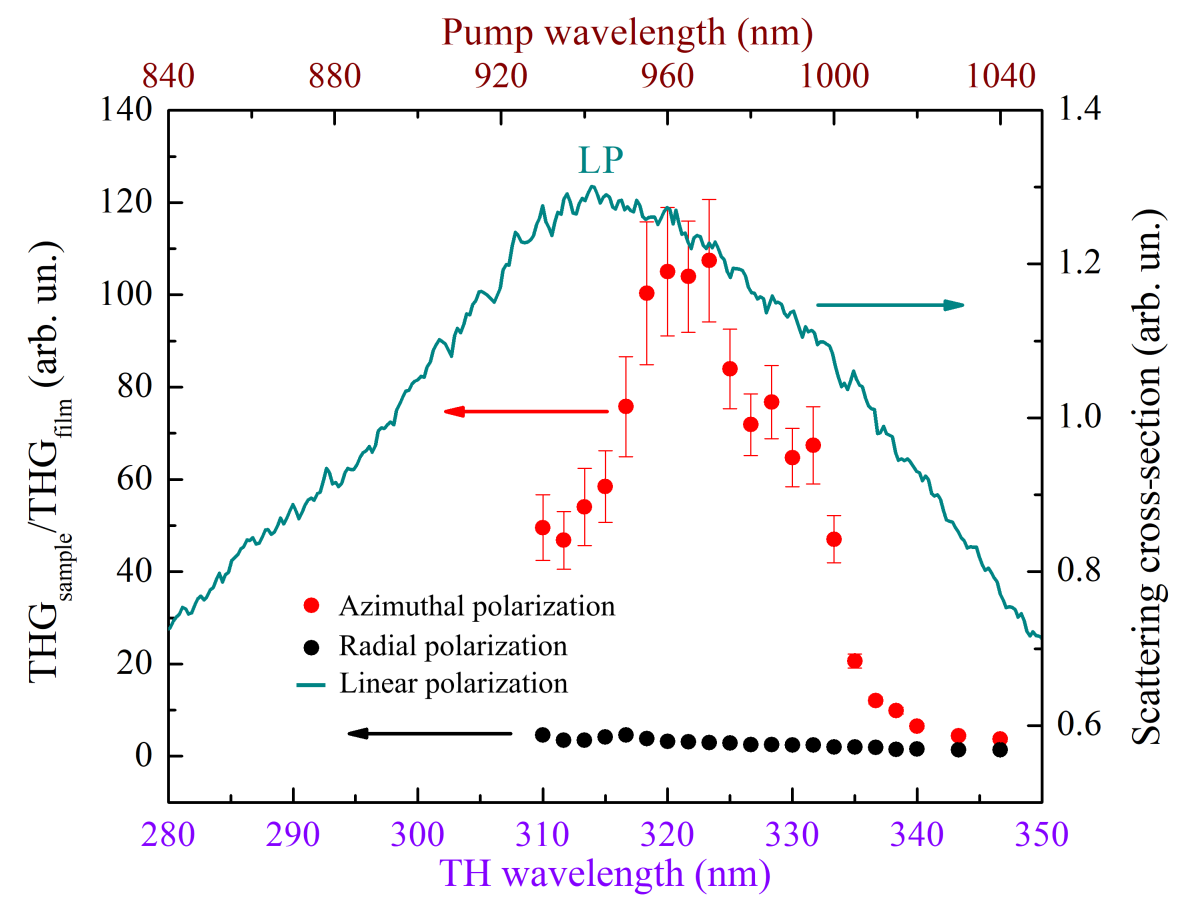

Figure S11. Green line shows scattering cross-section spectrum for the trimer of silicon nanodisks with fixed geometrical parameters $(h=260 \pm 5 \mathrm{~nm}, d=270 \pm 6 \mathrm{~nm}, s=$ $87 \pm 6 \mathrm{~nm}$ ) for LB illumination at normal incidence. Dots shows the TH signal from the trimer of Si nanodisks with fixed geometrical parameters divided to the $\mathrm{TH}$ signal from an unstructured Si film for $\mathrm{AB}$ (red dots) and RB (black dots) illumination at normal incidence.

between the opposite parts of the beam [2]. Direct plane image of $\mathrm{AB}$ is visualized on a CMOS camera located directly behind the LCP (Fig. S10(a)). The beam has a characteristic intensity distribution presented in Fig. S10(a). For further characterization of the beam quality, a Glan-Taylor polarizer (GTP) is placed right behind the LCP as an analyzer. GTP is mechanically rotated with a step of $\pi / 18 \mathrm{rad}$, and the direct image of the beam is obtained on the camera for each rotational angle. In Fig. S10(b) results for $0, \pi / 4$, 
$\pi / 2$, and $-\pi / 4$ GTP angle are presented. Sections of the beam are symmetrically located with respect to the center of the beam, which indicates that the obtained polarization distribution is indeed azimuthal [2]. Red arrows indicate the electric field polarization in the area under study.

We have also carried out scattering spectroscopy and the TH micro-spectroscopy for a trimer with fixed geometrical parameters ( $h=260 \pm 5 \mathrm{~nm}, d=270 \pm 6 \mathrm{~nm}, s=87 \pm 6 \mathrm{~nm}$ ), the results are presented in Figure S11. In the scattering cross-section measured for the plane wave illumination and the electric field polarization parallel to the $\mathrm{x}$-axis of the oligomer, the MDR (resonance at $\lambda=940 \mathrm{~nm}$ ) is observed as in the quadrumer case. In nonlinear experiments, the THG enhancement is obtained in spectral vicinity of MDR for AB pumping. The TH intensity is approximately an order of magnitude larger comparing to unstructured a-Si film. For RB illumination of the isolated trimer, as it was expected, the THG tends to zero value.

Section 5: multipolar decomposition of the incident radiation. Multipolar decomposition of the incident radiation in terms of the vector spherical harmonics has the following form:

$$
\begin{aligned}
& \mathbf{E}_{\mathrm{inc}}=E_{0} \sum_{l, m}\left\{A_{M}(l, m) \mathbf{X}_{l m}(\theta, \varphi) j_{l}(k r)-\frac{i}{k} A_{E}(l, m) \nabla \times\left[j_{l}(k r) \mathbf{X}_{l m}(\theta, \varphi)\right]\right\}, \\
& \mathbf{H}_{\mathrm{inc}}=\frac{i}{k} \nabla \times \mathbf{E}_{i}=E_{0} \sum_{l, m}\left\{A_{E}(l, m) \mathbf{X}_{l m}(\theta, \varphi) j_{l}(k r)+\frac{i}{k} A_{M}(l, m) \nabla \times\left[j_{l}(k r) \mathbf{X}_{l m}(\theta, \varphi)\right]\right\} .
\end{aligned}
$$

Here $A_{E, M}(l, m)$ are multipolar coefficients of the incident radiation, $\mathbf{X}_{l m}(\theta, \varphi)$ are the vector spherical functions, $j_{l}(k r)$ is the spherical Bessel function of order $l$, the wave number $k=\omega / c$. Using orthogonality relations of the spherical functions, we express multipolar coefficients through the surface integrals of scalar multiplication of the vector spherical harmonics with electric or magnetic fields of the incident radiation [3]:

$$
\begin{aligned}
A_{E}(l, m) & =\frac{\iint \mathbf{H}_{\mathrm{inc}} \mathbf{X}_{l m}^{*}(\theta, \varphi) d S}{E_{0} r^{2} j_{l}(k r)}, \\
A_{M}(l, m) & =\frac{\iint \mathbf{E}_{\mathrm{inc}} \mathbf{X}_{l m}^{*}(\theta, \varphi) d S}{E_{0} r^{2} j_{l}(k r)},
\end{aligned}
$$

where $d S=r^{2} \sin \theta d \theta d \phi$. These expressions can be rewritten through the angular functions $\pi_{l m}(\theta)$ and $\tau_{l m}(\theta)$ defined in Ref. [3]:

$$
\begin{aligned}
& A_{E}(l, m)=\frac{i O_{l m}}{E_{0} r^{2} j_{l}(k r)} \iint\left(H_{\mathrm{inc} \theta} \pi_{l m}-i H_{\mathrm{inc} \phi} \tau_{l m}\right) d S \\
& A_{M}(l, m)=\frac{i O_{l m}}{E_{0} r^{2} j_{l}(k r)} \iint\left(E_{\mathrm{inc} \theta} \pi_{l m}-i E_{\mathrm{inc} \phi} \tau_{l m}\right) d S .
\end{aligned}
$$

We use the following definition of an azimuthally polarized cylindrical vector beam 
(AP) [4] in our numerical modeling:

$$
\begin{aligned}
& \mathbf{E}_{\mathrm{inc}}=\hat{\varphi} E_{0} 2 \int_{0}^{\alpha_{M}} \sqrt{\cos \alpha} \sin \alpha l_{0}(\alpha) J_{1}\left(\varkappa_{\perp} \rho\right) e^{-i k_{||} z} d \alpha \\
& l_{0}(\alpha)=\exp \left(-\left(\beta_{0} \frac{\sin \alpha}{\sin \alpha_{M}}\right)^{2}\right) J_{1}\left(2 \beta_{0} \frac{\sin \alpha}{\sin \alpha_{M}}\right)
\end{aligned}
$$

where $l_{0}(\alpha)$ is the apodization function, $J_{1}\left(\varkappa_{\perp} \rho\right)$ denotes a Bessel function of the first kind,

of order $1, \rho=\sqrt{x^{2}+y^{2}}, k_{h}=\frac{2 \pi n_{h}}{\lambda_{0}}, \varkappa_{\perp}=k_{h} \sin \alpha, k_{\|}=k_{h} \cos \alpha, \alpha_{M}=\arcsin \frac{N A}{n_{h}}, \beta_{0}$ is the ratio of the pupil radius and the beam waist, $N A$ is a numerical aperture.

AP beam consists of magnetic multipoles with azimuthal index $m=0$. After numerical integration we obtain the following relations of magnetic multipolar contributions in the electromagnetic field of AP beam with parameters $\lambda_{0}=940 \mathrm{~nm}, \beta_{0}=3 / 2, N A=0.85$, $n_{h}=1.22$ :

$$
\left|\frac{A_{M}(2,0)}{A_{M}(1,0)}\right|=2.2, \quad\left|\frac{A_{M}(3,0)}{A_{M}(1,0)}\right|=3.6
$$

Section 6: Multipolar decomposition of eigenmodes. The optical eigenmodes of the quadrumer are calculated with the 3D FEM eigenmode solver in COMSOL. Once the internal field in the nanoparticles is obtained from numerical simulations, polarization currents are expanded into a series of spherical harmonics following Ref. [5]. The multipolar coefficients are then used to evaluate their partial contributions in the total radiation. The resultant percentage compositions of the modes are shown in Fig. 2(d) of the main text.

\section{References}

[1] Q. Zhan, "Cylindrical vector beams: from mathematical concepts to applications," Advances in Optics and Photonics 1, 1-57, 2009.

[2] M. Stalder and M. Schadt, "Linearly polarized light with axial symmetry generated by liquid-crystal polarization converters," Optics Letters 21, 1948-1950, 1996.

[3] C. F. Bohren and D. R. Huffman, Absorption and scattering of light by small particles, John Wiley \& Sons, 2008.

[4] K. S. Youngworth and T. G. Brown, "Focusing of high numerical aperture cylindricalvector beams," Optics Express 7, 77-87, 2000.

[5] P. Grahn, A. Shevchenko, and M. Kaivola, "Electromagnetic multipole theory for optical nanomaterials," New Journal of Physics 14, 093033, 2012. 